\title{
El rito de iniciación en La philosophie dans le boudoir del Marqués de Sade: tiempo hierático e isolismo
}

\author{
Berenice ORTEGA VILLELA \\ Universidad Nacional Autónoma de México
}

Proyectada mucho antes de la explosión revolucionaria y publicada hasta 1795 , cuando Sade se encontraba libre después de haber pasado doce años de encierro en la Bastilla, La philosophie dans le boudoir es una de las tres novelas en las que el Marqués desarrolla los elementos del mundo narrativo perverso que lo ha trascendido. La nouvelle Justine ou les infortunes de la vertu, suivie de Juliette, sa sœur y la inconclusa Les 120 journées de Sodome completan la tríada de textos en los cuales se desarrolla lo que hemos llamado, de manera errónea,"sadismo": el placer derivado de ejercer violencia sobre los otros. Dicho término, utilizado tanto por expertos como no para hablar de los temas de Sade no sólo es problemático por el hecho de que crea ideas preconcebidas acerca de lo que encontramos al enfrentarnos al texto sadeano, sino también porque deja de lado el término que el Marqués mismo creó para definir su concepción del ser en el mundo: el isolismo. Un poco olvidado de los estudios sadeanos, este término es producto de la experiencia en prisión de Sade y encierra una complejidad idéntica a la construcción de los relatos que lo desarrollan. Este trabajo busca establecer la relación entre isolismo, encierro y temporalidad, a partir de algunos conceptos de la triple mimesis de Paul Ricœur y del análisis de la estructura de La philosophie dans le boudoir.

PALABRAS CLAVE: temporalidad, isolismo, iniciación, ritualidad, libertinaje, sexualidad, siglo XVIII, literatura libertina.

La philosophie dans le boudoir written during Sade's twelve-year imprisonment in La Bastille, but published after his release in 1795, is one of the three novels (La nouvelle Justine ou les infortunes de la vertu, suivie de Juliette, sa sœur and the unfinished Les 120 journées de Sodome) in which the Marquis expands the components of the deviant narrative world that has transcended him. In this trilogy, the concept of what has come to be known as sadism (the pleasure originated from applying violence onto others) is developed. However, this term, although used by experts and amateurs alike to discusssadean themes, is a misnomer and problematic. This is because it not only creates a variety of prejudices about what the reader may encounter in the text, but also because it disregards the concept that the Marquis himself created to name his concept of Being (Sein) in the world: isolism, a term commonly forgotten in Sadean studies even though it is one that originates Sade's experience in prison and has a complexity only matched by the structure of the stories that surround the concept itself. This article establishes the relation between isolism, imprisonment, and temporality 
based on Paul Ricœur's ideas of triple mimesis and the structural analysis of $\mathrm{La}$ philosophie dans le boudoir.

KEYWORDS: temporality, isolism, bildungsroman, ritual, debauchery, sexuality, XVIII century, libertine literature.

El tema central de La philosophie dans le boudoir es la iniciación libertina de una joven de quince años, o, en palabras de Mme. de Saint-Ange, la iniciación de Eugénie "dans les plus sécrets mystères de Vénus" (12). El tópico de iniciación es recurrente en las novelas de la tradición libertina, ${ }^{1}$ pero en su texto, Sade "réinterprète, parodie [et] extrait la substance imaginaire de la situation intiatique" (Reichler, 1982: 102) para trasladarla al plano de lo sagrado. Este desplazamiento, de lo profano a lo ritual, implanta en el texto una temporalidad que permite invertir valores morales y levantar prohibiciones sociales. La trama de la historia es sencilla; a lo largo de ella, Eugénie y también los lectores de Sade se involucran en una clase teórico-práctica donde se desglosan los preceptos del libertinaje extremo ejercido no sólo por Saint-Ange y Dolmancé, sino también por otros villanos del mundo narrado del autor. El orden de exposición de los temas por aprender la clase es gradual y las disertaciones alrededor de éstos versan, a decir de Foucault, sobre cuatro constataciones de inexistencia que resumen el contenido de lo que en un inicio podríamos llamar la "filosofía libertina" de los villanos creados por el Marqués:

Dieu n'existe pas, et la preuve que Dieu n'existe pas c'est qu'il est entièrement contradictoire [...] Deuxième constatation: l'âme n'existe pas elle non plus, parce qu'elle est contradictoire $[\ldots]$ Troisième constatation d'inexistence: le crime n'existe pas [...] Quatrième constatation d'inexistence, la nature n'existe pas ou plutôt la nature existe mais si elle existe, ce n'est jamais que sur le mode de la destruction et par conséquent de la suppression de soi-même (La grande étrangère. À propos de littérature, 2013: 177-179).

Las acciones que conducen a Eugénie al libertinaje son la afirmación corporal del poliedro de cuatro caras formado por la teoría y se sitúan siempre en un vaivén entre la transgresión moral y la sexual: Eugénie primero niega la existencia de Dios, luego se vuelve sodomita, después pierde la virginidad y, por último, como concreción de su aprendizaje, comete incesto al participar en la violación y tortura colectivas de su madre.

\footnotetext{
${ }^{1}$ Más allá de la ficción, en el campo de las ideas la educación es un tema fundamental en las discusiones de los filósofos de la Ilustración. La educación de las jóvenes es, además, específico dentro del debate. En ese sentido, en 1783 la Academia de Châlons-sur-Marne propuso un debate público en torno a la pregunta "Quels seraient les meilleurs moyens de perfectionner l'éducation des femmes?", al cual Pierre Choderlos de Laclos contestó con el texto Traité sur l'éducation des femmes. Debido a las fechas de publicación es posible pensar que La Philosophie es un intento de Sade por responder también a dicha convocatoria desde la ficción.
} 
La trama y los agentes que contribuyen al desarrollo de las acciones son claros, más no así el género textual y la estructura del mismo. Si se deja a un lado la brusca interrupción del panfleto "Français, encore un effort si vous voulez être républicains", el diálogo entre los personajes transcurre con base en una progresión de las acciones: la presentación de un proyecto de educación, el aprendizaje paso por paso del libertinaje mental y físico y la ordalía final que convierte a Eugénie en libertina.

Desde esta apreciación superficial, parecería que en el proyecto de escritura de $L a$ philosophie dans le boudoir existe sólo la intención de contar una historia de iniciación, es decir, hay un hecho aislado, base de la construcción del mythos. ${ }^{2}$ Pero ¿por qué construir una trama a través de diálogos y no exponerla por medio de un narrador?, ¿por qué incluir en ellos didascalias si no hay una intención de puesta en escena?, ¿qué relación existe entre la trama y la configuración de las acciones? y ¿por qué interrumpir el diálogo con la intromisión de un extenso panfleto, cuya voz narrativa se desconoce, pero parece cercana a los actantes de la historia?

Para dar respuesta a las dos preguntas iniciales es necesario establecer, primero, qué entendemos por novela en el siglo XVIII y qué entendía el mismo Sade de este género. Así, una vez aclarada su intención en cuanto a la configuración, será posible avanzar hacia una interpretación del contenido para responder a los cuestionamientos ulteriores.

\section{Entre la novela y el tratado filosófico}

En Le roman au XVIII siècle, Françoise Barguillet recorre distintas definiciones de autores de la época y concluye que en el siglo XVIII podemos admitir como novela:

[...] Un écrit en prose que réponde au projet de raconter une histoire évoluant vers un dénouement: s'il n'y a qu'échange d'idées au cours d'un dialogue, comme dans $L e$ neveu de Rameau, nous ne pouvons user de cette dénomination. La fiction doit y supplanter la part (inévitablement dans toute création) de souvenirs personnels à l'auteur: si celui-ci, à l'instar de Rousseau dans les Confessions ou les Rêveries, annonce un dessein autobiographique, nous ne considérons pas qu'il y a là un roman. Enfin il fait vivre des personnages bien individualisés dont la silhouette se découpe souvent sur une toile de fond brossé en quelques touches puissants, qu'il s'agisse de la société du temps ou de certains sites. Néanmoins il n'est pas nécessaire que cet univers représente le monde réel: il peut obéir à d'autres lois, ce qui nous conduit à accepter comme romans les contes fantaisistes. Les récits oraux appelés "contes" et les "histoires" sont eux aussi admis dans la mesure où ils correspondent aux caractéristiques précitées (1981: 10).

\footnotetext{
${ }^{2}$ Tomo el término en el sentido en que lo utiliza Ricœur en Tiempo y narración: construcción de la trama, el qué de la mimesis.
} 


\section{$114 \square$ EL RITO DE INICIACIÓN EN LA PHILOSOPHIE DANS LE BOUDOIR DEL MARQUÉS DE SADE}

En aras de la practicidad, el autor engloba en una misma definición relatos cortos - historia, cuento, nouvelle - y extensos, con la intención de no inmiscuirse en disputas inútiles generadas por la diversidad de títulos y formas de las expresiones literarias de la época que comparten la intención de contar una historia. Para Barguillet, la forma no representa un problema tanto como el contenido, por ello, divide la novela dieciochesca en tres grandes vertientes de acuerdo con sus temas: novela romanesque, novela realista y novela filosófica, última categoría donde ubica a los textos sadeanos.

La definición de Barguillet se basa, entre otras, en la del propio Sade, quien hace un recorrido histórico de la novela en Idée sur les romans. Desde la Antigua Grecia hasta algunos de sus contemporáneos, Sade explica las características formales del género y establece una postura personal respecto a la creación. De acuerdo con él: "on appelle roman, l'ouvrage fabuleux composé d'après les plus singulières aventures de la vie des hommes" (1801: 97). Además, para el Marqués el origen de la novela está en la concepción mítica del mundo, ya que en ésta prevalece el interés de los hombres por entender y asimilar lo incomprensible: “À peine les hommes eurent-ils soupçonné des êtres immortels, qu'ils les firent agir et parler, des lors voilà des métamorphoses, des fables, des paraboles, des romans, en un mot, voilà des ouvrages de fiction, dès que la fiction s'empare de l'esprit des hommes" (102).

La creencia en un ser supremo es universal en los hombres, tanto como el sentimiento amoroso, por ello, las primeras obras de ficción "peignirent les objets fabuleux de son culte, tantôt ceux plus réels de son amour" (102). Aunque con esto Sade infiere la existencia de la novela desde los griegos, más adelante afirma que el primer y verdadero novelista es Cervantes y El Quijote "doit se considérer comme le premier de tous les romans [qui] possède sans doute plus qu'aucun d'eux, l'art de narrer, d'entremêler agréablement les aventures, et particulièrement d'instruire en amusant" (104). En la concepción poética de Sade, el arte de narrar está en el talento del autor para hilar historias capaces de divertir, pero también de educar. Asimismo, aclara que el arte de narrar no consiste en apegarse a una serie de reglas, sino en el ingenio del autor para imitar las acciones de los hombres, las cuales no tienen por qué ser verdaderas, sino únicamente verosímiles dentro del mundo narrado: "On ne te demande pas d'être vraie, mais seulement d'être vraisemblable" (125). Dicha verosimilitud no es un freno de la imaginación, sino únicamente lo que permite que "le dénouement [soit] tel que les événements le préparent, que la vraisemblance l'exige, que l'imagination inspire” (128).

Las ideas de Sade a propósito de la creación están cerca del pensamiento de Aristóteles, quien aprecia la originalidad de la creación y posiciona a la mimesis (imitación de la realidad) como sostén de la obra de arte. Esta concepción se aleja, a su vez, de los exégetas renacentistas italianos — mucho más cercanos, en tiempo, a Sade — quienes instauran la rigidez en las normas estilísticas, después defendida por Boileau y las subsecuentes corrientes literarias francesas. Por ello, con base en estas consideraciones, es posible afirmar que en las reflexiones poéticas de Sade se vislumbra un deseo de regresar a los textos fundadores del arte occidental para distinguirse de las concepciones artísticas de sus contemporáneos. 
De acuerdo con las definiciones de Barguillet y Sade, si la característica fundamental de la novela en el siglo XVIII es, de manera exclusiva, la intención de ficción, entonces se puede afirmar que La philosophie dans le boudoir es una novela. Sin embargo, dicha aseveración puede ser prematura y parcial sin antes cuestionar los elementos de otros géneros identificados en la forma textual.

Para Béatrice Didier, La philosophie dans le boudoir "ne s'agit pas d'un roman" (1972: 69) sino de un texto "à mi-chemin entre le traité philosophique et le roman" (59) o incluso "à mi-chemin entre le dialogue philosophique pur et une véritable pièce de théâtre" (65). En su artículo, Didier no especifica qué entiende por novela, y su afirmación a propósito de la incertidumbre generada por el texto se aleja del pragmatismo de Barguillet sin ser del todo estéril, debido a que resalta el interés del autor por constituir de una y no de otra forma el texto. La clasificación de Didier también resalta el perfil filosófico de la narración, e incluso preconiza dicha característica. Con ello, la autora parece no proporcionar demasiada importancia al hecho de que el tratado filosófico y la novela se distinguen por una característica esencial: la intención o no del relato de ficción. Un tratado filosófico no narra una historia, ya sea ficticia o verdadera, expone y desarrolla los principios de una filosofía, producto de un método. Por el contrario, una novela, sin distinguir su género, relata acciones ficticias. Aunque pareciera ocioso llevar a cabo estas precisiones en la terminología, considerar que La philosophie dans le boudoir tiene tintes de tratado filosófico puede llevar a conclusiones erróneas en el análisis literario. Es cierta la existencia de una intención filosófica en el texto, como la hay en una gran parte de los textos literarios, pero ésta se subordina siempre a la del relato. Por ello, los discursos y las acciones deben juzgarse siempre bajo la égida de la ficción.

En su artículo, Didier también se pregunta por los motivos de Sade para preferir la forma dialogada de la narración al tratado teórico, y se responde que en dicha intención ve: "A la fois un désir de convaincre, une sorte de souci apostolique, mais aussi une manifestation de la volonté de puissance: l'écrivain se satisfait de pousser l'adversaire idéologique dans ses derniers retranchements et de le tenir à sa merci” (60). En esta primera postura hay una tendencia a adjudicar los discursos de los personajes al escritor; de asumir que la intención de Sade es moralizar a sus lectores e imponer una filosofía. Sin embargo, a pesar de que las consideraciones preliminares de Didier respecto a la forma textual la llevan por el camino de la filosofía, sus conclusiones se acercan más al terreno de la mimesis. De manera acertada, aunque no lógica con su exposición, Didier enfatiza que: "Chez Sade [...] le dialogue est parfaitement clos, comme l'enceinte du château ou comme le boudoir où il se déroule. Seul y triomphe la voix du libertin, tandis que celle de l'adversaire est faible, dérisoire" (60) y con ello pone en evidencia la trampa textual de la forma: Sade no intenta comunicar y dialogar con el lector, sino imponer. En el texto sadeano hay, entonces, el deseo de comunicar no sólo a través del contenido, sino también de la forma. El diálogo es una herramienta de la ficción para sumergir al lector en el espacio narrativo, no un instrumento para exponer un sistema filosófico. 
Al leer La philosophie dans le boudoir no asistimos al intercambio de ideas entre distintos hablantes, sino a la afirmación reiterada de los cuatro postulados de inexistencia de los que habla Foucault. Así como al boudoir de Mme. de Saint-Ange únicamente ingresan quienes sirven a los propósitos del libertinaje, en la conversación sólo se admiten intervenciones de aquellos que concuerdan con los cuatro postulados (por ello el Caballero de Mirveil es silenciado en cuanto intenta distanciarse de los absolutos de Dolmancé). Esta idea se confirma en la dedicatoria “Aux libertins" que precede al relato:

\begin{abstract}
Voluptueux de tous les âges et de tous les sexes, c'est à vous seuls que j'offre cet ouvrage; nourrissez-vous de ses principes, ils favorisent vos passions [...] n'écoutez que ces passions délicieuses, leur organe est le seul qui doive vous conduire au bonheur $[\ldots]$ Femmes lubriques, que la voluptueuse Saint-Ange soit votre modèle [...] Jeunes filles trop longtemps contenues dans les liens absurdes et dangereux d'une vertu fantastique et d'une religion dégoutante, imitez l'ardente Eugénie [...] Et vous aimables débauchés, vous qui, depuis votre jeunesse, n'avez plus d'autres freins que vos désirs, et d'autres lois que vos caprices, que le cynique Dolmancé vous serve d'exemple (1998: 3).
\end{abstract}

El texto busca una afirmación en las mujeres lascivas, las jóvenes reprimidas por la religión y los depravados. Así, los personajes principales deben ser vistos como modelos a seguir, héroes ejemplares que guiarán las acciones de los lectores. En este sentido, la construcción del texto se encuentra muy lejos del modelo dialógico de la novela (tal como lo entiende Bajtin en sus textos sobre la narrativa de Dostoievski) ${ }^{3}$ y se acerca más auna estructura monológica, reforzada por el hermetismo del espacio narrativo, cuya característica principal es el aislamiento. ${ }^{4}$

A pesar de que en la narrativa Sade prefiere la representación del diálogo ${ }^{5}$ dicha forma es sólo una trampa textual. En realidad, en La philosophie dans le boudoir las

\footnotetext{
${ }^{3}$ Es importante recordar que la concepción bajtiniana de la novela surge de los textos renacentistas en forma de diálogo que proliferaron en dicha época y en los cuales el pensador ruso observa un síntoma de la modernidad, derivada de los cambios de pensamiento. Sade, al crear diálogos cerrados, parodia la tradición renacentista, pues invierte la diversidad de ideas representada en el dialogismo al unificar la voz de los distintos hablantes en un discurso único e irrefutable.

${ }^{4}$ En el interior del boudoir sólo son aceptados los discursos que reafirman la "filosofía libertina". Únicamente dos voces del exterior son escuchadas: la primera se materializa a través del panfleto "Français encore un effort si vous voulez être républicains", que interrumpe el hilo narrativo, enfrenta a los personajes con la Historia (la propuesta que se hace mediante el panfleto se enfoca en solucionar el conflicto posrevolucionario concerniente a la religión y a la forma de gobierno) y sitúa al lector en una posición de tensión provocada por tiempos verbales del presente. La segunda voz pertenece al padre de Eugènie, quien por medio de un mensaje legitima las enseñanzas desde su posición familiar, al externar que Dolmancé y SaintAnge "[auront] un peu travaillé pour [lui]" al pervertir a su hija (164).

${ }^{5}$ En la estructura de Justine y Juliette también el diálogo es preponderante, ya que, a pesar de que en la primera hay un narrador en tercera persona y en la segunda uno en primera, ambos sujetos narrativos reportan siempre de forma literal e inverosímil los largos discursos "filosóficos" de los libertinos que participan en la historia. Con esta técnica Sade crea una puesta en abismo en donde el origen-yo de la narración se disuelve en otros sujetos narrativos.
} 
conversaciones que se recrean están cerradas pues no hay en ellas un contrapeso capaz de generar un cambio de ideas. Existe, pues, una discordancia entre la forma y su contenido. La recreación del diálogo es una actividad mimética en primer grado, pues en ella no existe un narrador susceptible de sospecha; además, en el diálogo el lector se convierte en espectador e incluso partícipe tácito de la conversación. La forma dialogada sitúa al texto en el ámbito de lo dramático, pero también en el del comentario. ${ }^{6}$ El tiempo del discurso es diferente del tiempo de la narración: "el comentario se caracteriza por la tensión o el compromiso, la narración, por la distensión o relajación" (Ricœur, 1985: 480). A cada situación del lenguaje pertenecen distintos usos de los tiempos verbales del francés, en el comentario se pondera el presente, el pasado compuesto y el futuro; en la narración se usan los tiempos del imperfecto, el pasado simple, el pluscuamperfecto y el condicional, así: "Lo que los gramáticos llaman el pasado y el imperfecto [...] son tiempos de la narración, no porque la narración exprese fundamentalmente acontecimientos pasados, reales o de ficción, sino porque estos tiempos orientan hacia una actitud de distensión. Lo esencial es que el mundo narrado es extraño al entorno directo e inmediato del hablante y del oyente" (481).

A pesar de que en la lectura de La philosophie dans le boudoir las afirmaciones e imperativos nos conducen siempre al mismo lugar (la instauración de un modelo ejemplar de conducta y la aceptación de una "filosofía del crimen"), el propio texto genera un desconcierto en el lector, provocado por la forma textual y los tiempos verbales dominantes en el relato.

Al leer la Philosophie dans le boudoir estamos preparados para sumergirnos en la ficción, para experimentar y asimilar también la distentio animi de san Agustín retomada por Ricœur en su exposición de la triple mimesis, pero en lugar de eso, encontramos tensión discursiva, la cual, en efecto, no hace más que acorralarnos. Los diálogos recreados por Sade son monólogos desde la soledad del encierro; como dice Didier: "Tout l'art, toute la technique de Sade consiste donc non pas tant à le vaincre [l'adversaire intellectuel], ce qui est trop facile, mais en faire durer la joute" (1972: 63). De tal forma, podemos decir que su técnica discursiva consiste en juntar forma $y$ contenido. El aislamiento del boudoir, en donde sólo se permite la entrada de locutores afines al discurso que se expone es reflejo del diálogo cerrado que repite al infinito afirmaciones absolutistas e imperativas.

\footnotetext{
${ }^{6}$ De acuerdo con Käte Hamburger: "Una barrera infranqueable separa el discurso asertivo, que versa sobre la realidad, del relato de ficción. De este corte se deriva una lógica diferente [...]. La razón de esta diferencia $[. .$.$] proviene de que la ficción remplaza al origen-yo del discurso asertivo, que es real, por el$ origen-yo de los personajes de ficción" (cita en Ricœur, Tiempo y narración II, 476). Además "lo épico abarca todo el campo narrativo; lo dramático, el de la acción llevada a la escena por personajes que dialogan ante el espectador, y lo lírico, la expresión por la poesía de los pensamientos y sentimientos que experimenta el escritor. De esta forma, sólo pertenece a la ficción el género épico [...] y el dramático" (475). De tal forma, es posible decir que el discurso asertivo no es ficción en tanto que utiliza verbos en presente; sin embargo, los personajes que dialogan en un presente de la narración, están en un tiempo ficticio.
} 
Además de la influencia del espacio cerrado en el desarrollo de la trama, la técnica de Sade consiste también en alternar la tensión del discurso con la distensión de la acción. Si se observa con detalle la construcción de los diálogos, aquéllos en los que se desarrollan los conceptos del libertinaje son más extensos y cargados; en ellos el discurso se expone mediante largos párrafos, compuestos por oraciones subordinadas que apenas permiten un respiro al lector. Por el contrario, en la descripción de la orgía, las oraciones son breves y entrecortadas, lo cual desemboca en una lectura ágil. La alternancia entre saturación del discurso y repetición de la orgía provoca, además, un desvanecimiento de la temporalidad. ${ }^{7}$ El primer diálogo comienza con una indicación temporal y el último cierra la narración con otra referencia al tiempo oficial; ${ }^{8}$ pero en el transcurso de la lección esas referencias desaparecen por completo. En el proceso iniciático, el tiempo oficial marcado por las horas o los horarios de los alimentos en la cultura francesa no tiene cabida.

No se puede obviar la intención pedagógica de la forma dialogada. Así como los personajes tradicionales de la novela francesa pretenden educar a sus lectores e inclinarlos hacia determinada sensibilidad, la novela de Sade busca reeducar a los lectores de fin de siglo por medio de una nueva concepción de la experiencia estética. A través de Dolmancé y Saint-Ange, Sade intenta conmover más que educar moralmente. Eugénie, como personaje arquetípico, además de representar cierta educación sentimental, personifica al lector tradicional, educado dentro de una tradición estética conservadora del recato y las forma, y al cual Dolmancé y Saint-Ange pretenden destruir y reedificar. La intención moralizadora de los personajes se encuentra, evidentemente, inscrita en el plano de la ficción y coincide con el esquema narrativo, es decir, con la iniciación libertina de la joven. En Idée sur le roman, Sade aconseja a todo aquel que desea ser escritor: 'Évite l'afféterie de la morale; ce n'est pas dans un roman qu'on la cherche; si les personnages que ton plan nécessite sont quelquefois contraints à raisonner, que ce soit toujours sans affectation, sans la prétention de le faire, ce n'est jamais l'auteur

\footnotetext{
${ }^{7}$ No se indica la hora exacta en que los personajes llegan a la residencia de Saint-Ange. En el primer diálogo, el Caballero de Mirveil informa que Dolmancé "arrivera à 4 heures précises"; sin embargo, la llegada prematura del personaje en el tercer diálogo contradice dicha afirmación. En el mismo primer diálogo, el Caballero informa: "Nous ne dînons qu'à 7, nous aurons comme tu vois, tout le temps de jaser", a este respecto, Michel Delon anota que se puede inferir que la acción se llevará a cabo, de acuerdo con la tradición clásica, en un lapso no mayor a tres horas, puesto que, en efecto, el texto concluye con la orden de Dolmancé: "Nous mes amis [Dolmancé, Saint-Ange, Eugénie y Augustin], allons nous mettre à table, et de là tous quatre dans le même lit" (178). Por otro lado, antes de comenzar la lección, Eugénie informa "nous avons déjeuné à une lieue d'ici, j'attendrais maintenant jusqu'à 8 heures du soir sans éprouver le moindre besoin" (13), de lo que se puede inferir que la lección comienza al medio día, lo cual indicaría un lapso de la acción de más de cuatro horas (ver nota 1, página 5 de la edición Pléiade de $L a$ philosophie dans le boudoir).

${ }^{8}$ Tomo el término del análisis de La señora Dalloway que hace Ricœur en Tiempo y narración II: "es importante observar que el tiempo oficial al que los personajes se enfrentan no es sólo este tiempo de los relojes, sino también cuanto tiene de convivencia con él” (543). Más adelante se ahondará en la diferencia entre este tiempo oficial y el tiempo íntimo.
} 
qui doit moraliser, c'est le personnage, et encore ne le lui permet-on que quand il y est forcé par les circonstances" (127).

Los discursos de los personajes son, entonces, una herramienta del mythos y el lector debe ver en ellos un camino que conduce al objetivo último de la narración, no una intención del escritor de desarrollar una filosofía en torno al crimen.

Por lo tanto, La philosophie dans le boudoir no es un diálogo filosófico, tampoco una obra de teatro y mucho menos un tratado, se trata de una novela híbrida que conjunta todos esos géneros. ${ }^{9}$ De tal forma, las palabras utilizadas por Saint-Ange para describirse a sí misma la convierten en la personificación de la novela: "Enfin, mon cher, je suis un animal amphibie, j'aime tout, je m'amuse de tout, je veux réunir tous les genres" (5). La construcción en diálogos, la utilización de didascalias e incluso la irrupción de un panfleto dentro del hilo narrativo son recursos para reforzar una estructura que, en sí misma, es burla de las tradiciones renacentista y clásica. La hibridación, como mezcla de dos cosas pertenecientes a una naturaleza distinta, constituye el primer desconcierto al que se enfrenta el lector y esa primera confusión es sólo el anuncio de las múltiples discordancias de la obra.

\section{Iniciación y experiencia temporal}

Para Ricœur "la configuración de la trama impone a la sucesión indefinida de los incidentes el sentido del punto final [...], aquél desde el que puede verse la historia como una totalidad" (1985: 135). De la comprensión de la historia como una conjunción significante, surge una nueva cualidad del tiempo, debido a que "al leer el final en el comienzo y el comienzo en el final, aprendemos también a leer el tiempo mismo al revés [...] el acto de narrar, reflejado en el de continuar una historia, hace productivas las paradojas que inquietaron a Agustín hasta el punto de llevarlo al silencio" (135).

De acuerdo con las consideraciones de Ricœur, la tortura de Mme. de Mistival es el clímax desde el cual debe considerarse el resto de la narración. A partir de esta perspectiva, las discordancias de la forma se acentúan, puesto que la sucesión de las acciones carece de verosimilitud, ya que cualquier lector avezado puede entrever que es improbable que una joven de quince años deseche en menos de un día una educación cristiana aprendida a lo largo de toda una vida. A propósito de la verosimilitud, en Idée sur le roman, Sade apunta que en toda novela "le dénouement doit être tel que les événements le préparent, que la vraisemblance l'exige, que l'imagination inspire" (128), por lo que no se le puede culpar de ignorancia respecto a este punto fundamental para la construcción de la trama. Incluso es clara la conciencia del escritor respecto a las transgresiones en sus historias, pues en el mismo ensayo se pregunta: "Ne

\footnotetext{
${ }^{9}$ En este sentido, sería pertinente analizar hasta qué punto podríamos hablar de una "novela teatral" de acuerdo con el término utilizado por Henrri Coulet en Études sur le roman francais au XVIII siécle.
} 
deviens-je pas doublement coupable de n'avoir pas bien fait, si je prouve que je sais ce qu'il faut pour faire bien?" (123).

¿Es posible, entonces, ver en la falta de verosimilitud un ardid del autor? A través del final, sabemos que en lugar de una historia de aprendizaje, las acciones en el texto recrean un ritual iniciático situado fuera del tiempo, cuyo propósito es ayudar a Eugénie a entrar en contacto con una propia naturaleza libertina, olvidada por la imposición social del cristianismo. El ritual que se materializa por medio de la repetición provoca una escisión entre el tiempo anterior y posterior, pues a través de la iniciación, Eugénie descubre a la naturaleza como fuerza motora del mundo y al cumplimiento del deseo como único paliativo del sufrimiento provocado por el caos existencial resultado de la desaparición de Dios.

Con la repetición continua de discursos de negación de los preceptos morales de la sociedad, y la descripción de una vorágine orgiástica, Sade transita del tiempo profano al sagrado y construye, con ayuda de algunos elementos característicos de los rituales primitivos, un rito iniciático libertino. Sin embargo, contrario a lo que sucede en los ritos iniciáticos tradicionales, en La philosophie dans le boudoir Eugénie pasa de lo colectivo a lo individual y descubre no la capacidad renovadora de la naturaleza, sino el caos reinante y la pulsión violenta del ser humano.

\subsection{Del tiempo profano al tiempo sagrado}

En su texto de 1958, Nacimiento y renacimiento. El significado de la iniciación en la cultura humana, Mircea Eliade revisa los significados y elementos simbólicos de los ritos iniciáticos de las culturas primitivas. De acuerdo con él, los ritos iniciáticos de las mujeres se diferencian de aquéllos de los hombres porque

[...] la iniciación femenina comienza con la primera menstruación. Este síntoma fisiológico, la señal de la madurez sexual, significa una ruptura: el alejamiento de la muchacha de su mundo familiar. Se la aísla de inmediato, separándola de la comunidad, algo que nos recuerda, en el caso masculino, la separación de la madre y la segregación $[\ldots]$ la duración de la segregación varía según las culturas, desde tres días $[\ldots]$ hasta veinte meses o incluso varios años [...] las muchachas conforman al final un grupo, y luego su iniciación se convierte en colectiva, bajo la dirección de las mujeres mayores de la familia (como en India) o de ancianas (África). Estas tutoras las instruyen en los secretos de la sexualidad y fertilidad, y les enseñan las costumbres de la tribu y al menos algunas de sus tradiciones religiosas, las accesibles a las mujeres. Así pues, la educación impartida es de carácter general, pero su esencia es religiosa; consiste en una revelación de la sacralidad de la mujer. Se prepara ritualmente a la muchacha para que asuma su modo de ser específico, es decir, para convertirse en creadora, y al mismo tiempo se le enseñan sus responsabilidades en la sociedad y en el cosmos, responsabilidades que, entre los pueblos primitivos, siempre son de naturaleza religiosa. 
Al hablar de los ritos de la pubertad masculinos hicimos referencia al complejo simbolismo del bosque y la cabaña; un simbolismo que es por una parte el del más allá, es decir el de la muerte, y el de la oscuridad de la gestación en el útero materno. El simbolismo de la oscuridad también se enfatiza en la segregación ceremonial de las muchachas, ya que son aisladas en un rincón oscuro de la casa, y entre muchos pueblos se las prohíbe ver el sol, un tabú cuya explicación radica en la conexión mística existente entre la luna y la mujer [...] En casi todas partes también existen, claro está, restricciones dietéticas, y entre algunos pueblos las novicias visten un atuendo especial [...] El elemento importante que sobresale es la segregación, que tiene lugar tanto en el bosque (como sucede entre los swahili), o bien en una cabaña al efecto [...] La ceremonia que concluye el proceso es tan esencial como la segregación, que constituye el primer rito iniciático (1958: 25).

En la descripción se advierte que el propósito de los ritos iniciáticos en las mujeres es dotar a las jóvenes de herramientas para asumir su función dentro de la sociedad. Esta función es religiosa, pues concierne a la capacidad procreadora; razón por la cual es común que el rito comience con la primera menstruación. En los ritos primitivos, la mujer, así como la Madre Naturaleza, representa la fertilidad y renovación constante del mundo. Todo rito constituye la incursión momentánea en un tiempo sagrado que se separa del tiempo profano porque en él se revelan conocimientos que permiten a los iniciados asumir su función en la sociedad y, en algunos casos, conocer el origen del mundo y del ser humano.

Por una parte, si se toma como base la descripción de Eliade, La philosophie dans le boudoir comparte con el rito primitivo al menos tres elementos simbólicos que construyen la distinción entre tiempo profano y tiempo sagrado. El primero de ellos es el aislamiento, pues, paralelo al rito primitivo, Eugénie es retirada de la tutela de su madre para acudir a la residencia de Saint-Ange (cuya ubicación se puede inferir en la campiña, lejos de la ciudad ${ }^{10}$ ), en donde pasará "deux jours délicieux" y de la cual no saldrá sin ser "savante". El segundo elemento es la restricción dietética, que no se impone a Eugénie pero ella misma asume cuando responde "je n'ai chère amie, d'autre besoin que celui de t'entendre" (13) a la invitación de Saint-Ange para desayunar antes de la lección. ${ }^{11}$ Finalmente, el tercer instrumento es el atuendo distintivo, materializado en las "simarres de gaze [qui] ne voileront [...] que ce qu'il faut cacher au désir" (15) que Saint-Ange proporciona a Eugénie y a Dolmancé antes de comenzar la enseñanza.

Por otra parte, igual que en el rito primitivo, a través de su iniciación, Eugénie obtiene la "revelación de la sacralidad de la mujer", pero ésta no se relaciona con el misterio de la procreación y la renovación del mundo (razón por la cual ni siquiera se

${ }^{10}$ Ver Michel Delon, Notice en Sade, Euvres, t. III. París: Pléiade.

${ }^{11}$ La afirmación de la joven, que dice no tener otra necesidad que escuchar a sus instructores, es eco de las palabras de Saint-Ange, cuando esta última afirmó, páginas antes, que Dolmancé y ella colocarían "dans cette jolie petite tête, tous les principes du libertinage le plus effréné, [ils l'embrasseront de leur] feu, l'alimenteront de [leur] philosophie et lui inspireront [leurs] désirs" (9). 
menciona el ciclo menstrual) sino con la inercia destructiva de la naturaleza y la consecuente anulación de la sacralidad de la procreación. Las obligaciones femeninas dentro de la sociedad libertina no son las de madre, vigilante y transmisora de costumbres, sino las de prostituta:

Mme. De Saint Ange: Dans quelque état que se trouve une femme, ma chère, soit fille, soit femme, soit veuve, elle ne doit jamais avoir d'autre but, d'autre occupation, d'autre désir que de se faire foutre du matin au soir. C'est pour cette unique fin que l'a créée la nature (39).

Sin embargo, la prostitución como imperativo y deber de la mujer no carece de religiosidad; el saber que se desarrolla durante la lección conduce a Eugénie a asumir el placer sexual como único objetivo de su existencia y a través del cual se puede dar sentido a la vida:

DolmANCÉ: Livrez-vous, Eugénie, abandonnez tous vous sens au plaisir, qu'il soit le seul dieu de votre existence; c'est à lui seul qu'une jeune fille doit tout sacrifier, et rien à ses yeux ne doit être aussi sacré que le plaisir (21).

La deificación del placer contribuye a la anulación de la capacidad procreadora de la mujer (columna vertebral, a decir de Rousseau en el Contrat Social, de la perpetuación de la familia como institución primigenia de la convivencia), y traslada de lo colectivo a lo individual la responsabilidad de ésta en la sociedad. En una sociedad regida por valores cristianos, el papel de la mujer en la colectividad es educar a los hijos y transmitir los valores morales; en la sociedad libertina, la mujer no debe ocuparse más que de su propio placer.

Como la intención primigenia del rito libertino es anular la reproducción femenina para servir a la inercia destructiva de la naturaleza, la pieza más importante de la tríada de elementos simbólicos que se mencionó, con base en lo dicho por Eliade, es el aislamiento. Los otros dos elementos (el atuendo y la restricción dietética) sólo se presentan de forma sutil para contribuir a la construcción de la diferencia entre un tiempo profano, que se desarrolla en el exterior, y uno sagrado, que transcurre en el boudoir. El tiempo profano queda entendido como el de la norma y la autoridad, el sagrado como el del relajamiento y lo hierático. El tiempo al interior del boudoir se vuelve sagrado en la medida en que "todo tiempo, cualquiera que sea, está 'abierto' sobre un tiempo sagrado, en otros términos puede revelar lo que llamaríamos con una fórmula cómoda lo absoluto, es decir lo sobrenatural, lo sobrehumano, lo sobrehistórico" (Eliade, 1964: 347); como ya se vio, a través de la iniciación del tiempo sagrado, Eugénie obtiene una revelación que atañe a lo absoluto, a su deber como individuo más allá de la sociedad. 
Ahora bien, en su texto de 1958, Eliade establece que el elemento primordial de los ritos iniciáticos de las sociedades secretas es el aprendizaje de un nuevo lenguaje que permite a los iniciados acceder a la sabiduría exclusiva compartida en ellas:

Todas las formas de iniciación de la pubertad, incluso las más elementales, implican la revelación de un secreto y de conocimientos sagrados. Algunos pueblos llaman "los conocedores" a sus iniciados. Además de las tradiciones tribales, los novicios aprenden una nueva lengua, que utilizarán más tarde para comunicarse entre ellos. Una lengua especial $\_\mathrm{o}$ al menos un vocabulario inaccesible para las mujeres y los no iniciados - es la muestra de un fenómeno cultural que se desarrollará totalmente en las sociedades secretas (28).

Los personajes libertinos de Sade dominan un lenguaje exclusivo que les permite transgredir la norma social, pues éste se relaciona con la liberación del cuerpo. En el primer diálogo, Saint-Ange pide a su hermano que describa los gustos de Dolmancé y el Caballero responde:

Le Chevalier: Tu le sais; les délices de Sodome lui sont aussi chers comme agent que comme patient; il n'aime que les hommes dans ses plaisirs, et si quelquefois néanmoins il consent à essayer des femmes, ce n'est qu'aux conditions qu'elles seront assez complaisantes pour changer de sexe avec lui. Je lui ai parlé de toi, je l'ai prévenu de tes intentions, il accepte, et t'avertit à son tour des clauses du marché. Je t'en préviens, ma sœur, il te refusera tout net, si tu prétends l'engager à autre chose: ce que je consens à faire avec votre sœur, est, prétend-il, une licence... une incartade dont on ne se souille que rarement et avec beaucoup de précautions.

Mme DE SAINT-ANGE: Se souiller !...des précautions! J'aime à la folie le langage de ces aimables gens; entre nous autres femmes, nous avons aussi de ces mots exclusifs qui prouvent comme ceux-là, l'horreur profonde dont elles sont pénétrées pour tout ce qui ne tient pas au culte admis... (Sade, 1998: 7).

En el "culto" libertino hay un rechazo por las costumbres sexuales del mundo profano y los "mots exclusifs" de los que habla Saint-Ange son pilares de la distinción entre vida social, cargada de prejuicios, y vida libertina, libre por completo de ellos. Por esto, no es gratuito que el rito iniciático de Eugénie comience por otorgar a la joven un nuevo léxico. De la misma forma en que el mundo se erige ante un niño a través de la adquisición de las palabras, la alumna de los instructores inmorales descubre, vocablo por vocablo, el universo corporal del libertinaje. Eugénie aprende el léxico utilizado en el macrocosmos y los nombres secretos del microcosmos: "ces globes de chair on les nomme indifféremment gorge, sein, tétons", "ce sceptre de Venus on le nomme membre", "ces mouvements se nomment pollution, et en terme de libertinage cette action s'appelle branler", "le mot technique est couilles,... testicules est celui de l'art", "une jolie fille ne doit s'occuper que de foutre et jamais d'engendrer", "cela s'appelle décharger" (16-20). 


\section{$124 \square$ EL RITO DE INICIACIÓN EN LA PHILOSOPHIE DANS LE BOUDOIR DEL MARQUÉS DE SADE}

A la par de la explicación de cada palabra y acción, Eugénie descubre, además, su corporeidad y su propio placer a través de la práctica. Ese placer, que, en palabras de la joven, es: "une sensation imposible à peindre" (22) es la clave de la "filosofía" que sus instructores están encargados de enseñarle.

\subsection{El isolismo al que estamos condenados}

La adquisición del lenguaje secreto del libertinaje y la experiencia de la sexualidad son el acceso a los conocimientos, o mejor dicho, a las verdades que fundamentan la emancipación del libertinaje. En todo momento, Sade juega con la verosimilitud de sus obras, en el prefacio de Justine y Juliette el autor ofrece su relato como salido de la realidad y no de la invención; asimismo, en la dedicatoria "Aux libertins" de la $L a$ philosophie dans le boudoir los personajes son presentados como modelos reales a seguir. Este juego es para Foucault el centro de la obra de Sade. Si en todo momento el escritor habla de la verdad de sus relatos, el lector debe entender por ésta:

[non pas] la vérité de ce qu'il raconte [mais] la vérité de ses raisonnements. Le problème du romancier au XVIII 'è siècle, c'était d'établir dans la forme de la vraisemblance, une fiction donc qui soit capable d'émouvoir, le problème de Sade est de démontrer une vérité $[. .$.$] Autrement dit, ce qui doit être vrai, c'est le raisonnement, c'est$ cette forme de rationalité qui est promue par l'exercice du désir ou qui soutient l'exercice du désir" (2013: 153).

La primera verdad de los razonamientos que se desarrollan en la lección, y de la cual se derivan los demás conocimientos de la educación de Eugénie, es la inexistencia de Dios:

EugÉNIE, à Mme de Saint-Ange: Quoi! réellement, mon aimable amie, l'existence de Dieu serait une chimère?

MmE DE SAINT-AngE: Et des plus méprisables, sans doute.

DOLMANCÉ: Il faut avoir perdu le sens pour y croire; fruit de la frayeur des uns et de la faiblesse des autres, cet abominable fantôme, Eugénie, est inutile au système de la nature (27).

Con la desaparición de Dios, el mito judeocristiano de la creación también se desvanece y en su lugar el libertino ofrece una explicación en la que la naturaleza es fuerza motora del mundo y los seres humanos: "il est démontré que l'homme ne doit son existence qu'aux plans irrésistibles de la nature" (27). La naturaleza como fuerza creadora no es un capricho del razonamiento libertino, sino un resultado del auge de las ciencias en la Ilustración. Los razonamientos de Dolmancé se fundamentan tanto en estudios antropológicos como en descubrimientos recién hechos de genética, así como en textos (un poco tergiversados) de filósofos como Rousseau y Voltaire, a quienes cita 
y discute. A través de sus fuentes y de acuerdo con el pensamiento de su tiempo, el libertino de Sade lleva al extremo la máxima de la Ilustración que ve en el ser humano el centro del universo y la clave para comprenderlo. ${ }^{12}$

El derrumbe de la figura de Dios como creador y regente de la conducta humana es el sustento de la nulidad de los valores cristianos y sociales (caridad, honestidad, recato sexual e incluso los crímenes en general: asesinato, robo, etcétera) que conduce, a su vez, a una conducta basada en las pulsiones. En el sistema del libertinaje, la palabra crimen pierde su significado porque: "la destruction, étant une des premières lois de la nature, rien de ce qui détruit ne saurait être un crime" (54) y en el lugar de los valores morales encarecidos por el cristianismo y las leyes, el libertino coloca al egoísmo como eje de las acciones humanas porque "rien n'est aussi indifférent sur la terre que d'y commettre le bien ou le mal; nous goûts, notre tempérament doivent seuls être respectés" (34).

Este retraerse en sí mismo y organizar el mundo a través de las propias pulsiones es el fundamento del isolismo. Aunque el término como tal sólo es utilizado en Justine y en Aline y Valcour, la enseñanza que se imparte a Eugénie gira en torno a este concepto. El isolismo define el aislamiento mental y físico de los personajes sadeanos y se puede entender en dos acepciones.

\subsection{El isolismo como abandono y su relación con la temporalidad}

Por una parte, en las primeras páginas de Justine, después de uno de los tantos infortunios del personaje, el narrador dice: "Et la malheureuse Justine, deux fois repoussé dès le premier jour qu'elle est condamnée à l'isolisme, entre dans une maison où elle voit un écriteau, loue un petit cabinet garni au cinquième, le paye d'avance et s'y livre à des larmes d'autant plus amères qu'elle est sensible et que sa petite fierté vient d'être cruellement compromise" (33).

El isolismo de Justine es el camino errático que significa su vida entera. A partir de la muerte de sus padres, la joven hermana de Juliette es arrojada al mundo y decide tomar el camino de la virtud para conducir su vida, pero en éste sólo encuentra vejaciones. En la oración "être condamné à l'isolisme" se concentra la realidad de Justine, cuyo destino parece no pertenecerle, puesto que, aunque siempre tome decisiones moralmente correctas, termina confiando en alguien que abusa de ella (Foucault, 2013: 156). El isolismo de Justine no atañe sólo a su soledad, también engloba el hecho de que en su errar por el mundo no encuentre a nadie que comulgue con sus valores; ni siquiera al mismo Dios, quien nunca intercede por ella cuando el horror la alcanza.

\footnotetext{
${ }^{12}$ Al respecto ver Ernst Cassirer, (1932), Filosofía de la Ilustración. México: FCE, y Viñas Piquer, (2002), Capítulo III. Humanismo y ciclo clasicista, en Historia de la crítica literaria, 2a. ed., Barcelona: Ariel.
} 
En esta primera acepción, el isolismo implica también un aspecto temporal. En el mundo narrado de Justine y también en el de La philosophie dans le boudoir, el ser humano es producto de un capricho del azar y se encuentra solo en el mundo, en constante lucha entre lo que la sociedad establece como norma y lo que la naturaleza dicta. En el mundo materialista ${ }^{13}$ que surge de la desaparición de Dios, el presente es la única ancla del ser humano en la realidad, puesto que tanto el devenir como el origen se han vuelto inciertos. En ese sentido, el isolismo es una intensificación de la experiencia temporal porque, de acuerdo con Ricœur, ésta

[...] no consiste sólo en pensar el tiempo como abolido bajo la perspectiva de la idea-límite de una eternidad que lo hiere con la nada. Tampoco se reduce a transferir al registro de la queja y del gemido lo que no era todavía más que un argumento especulativo. Intenta fundamentalmente extraer de la experiencia del tiempo recursos de jerarquización interna, cuyo provecho no será abolir la temporalidad sino profundizarla (79).

Para san Agustín la explicación de la inacción de Dios se encontraba en la perennidad del Verbo, el cual es punto de partida y retorno del ser humano. En Sade, el ser humano se encuentra privado de la estabilidad perenne y Dios queda reducido a un fantasma que atormenta en todo momento la psique de los personajes por su cualidad incomprensible.

Aunque en las disertaciones de los personajes de La philosophie dans le boudoir no se explicita de manera textual la cuestión temporal, ésta invade con profundidad las discusiones en torno al libertinaje. Para Dolmancé, la existencia de Dios no posee un sustento racional pues ${ }^{14}$

Ce Dieu, que les sots regardent comme auteur et fabricateur unique de tout ce que nous voyons, n'est que le nec plus ultra de la raison humaine, que le fantôme créé à l'instant où cette raison ne voit plus rien, afin d'aider à ses opérations [...] s'il est certain qu'à supposer que cet être inerte existât, ce serait assurément le plus ridicule de tous les êtres, puisqu'il n'aurait servi qu'un jour, et que depuis de millions de siècles il serait dans une inaction méprisable (27).

Como productos de la Ilustración, los personajes de Sade pasan todo su conocimiento del mundo tangible por el filtro de la razón y la ciencia, y el concepto de Dios se

${ }^{13}$ En el sentido desarrollado en L'homme machine de La Mettrie.

${ }^{14}$ Un razonamiento parecido es expuesto en el Dialogue entre un prêtre et un moribond: "«Vous ne croyez pas en Dieu?» demande le prêtre. «Non, répond le moribond. Et cela par une raison bien simple: c'est qu'il est parfaitement impossible de croire ce qu'on ne comprend pas [...] Je te défie toi-même de croire au Dieu que tu me prêches, parce que tu ne saurais pas me le démontrer, parce qu'il n'est pas en toi de me le définir, que par conséquent tu ne le comprends pas, que, dès que tu ne le comprends pas, tu ne peux plus m'en fournir aucun argument raisonnable, et qu'en un mot, tout ce qu'est au-dessus des bornes de l'esprit humain, est ou chimère ou inutilité; que ton dieu une pouvant être que l'une ou l'autre de ces choses, dans le premier cas je serais un fou d'y croire, un imbécile dans le second»" (11). 
alza frente a ellos como un límite del intelecto. Sin embargo, de manera paradójica, la reminiscencia constante de la inexistencia de Dios hace de éste una figura omnipresente, al grado que pareciera que el objetivo del rito orgiástico es obligar al fantasma a materializarse:

DolmancÉ: Un de mes plus grands plaisirs est de jurer Dieu quand je bande; il me semble que mon esprit, alors mille fois plus exalté, abhorre et méprise bien mieux cette dégoûtante chimère: je voudrais trouver une façon ou de la mieux invectiver, ou de l'outrager davantage, et quand mes maudites réflexions m'amènent à la conviction de la nullité de ce dégoutant objet de ma haine, je m'irrite, et je voudrais pouvoir aussitôt réédifier le fantôme, pour que ma rage au moins portât sur quelque chose (57).

En las palabras de Dolmancé, más que una constatación de inexistencia, parece haber un resentimiento de abandono (el mismo que sufre Justine) que se reitera en el momento culminante del rito iniciático, cuando Mme. de Mistival exclama "Juste ciel" y Dolmancé responde "N'implore pas, ma mie, il sera sourd à ta voix, comme il l'est à celle de tous les hommes; jamais ce Ciel puissant ne s'est mêlé d'un cul"' (171).

Así como Justine es condenada al isolismo: "le malheureux individu connu sous le nom d'homme, et jeté malgré lui sur ce triste univers" está condenado a sacrificar "tout à la volupté [pour] réussir à semer quelques roses sur les épines de la vie" (3, las cursivas son propias). El enunciado es curioso no sólo por su carácter categórico, sino también por su carácter fenomenológico. En él, Sade condensa el sentir que impregna su obra y que se sintetiza por medio del isolismo: el desamparo del ser humano que ha sido arrojado al mundo sin una guía moral.

Tal pareciera que para Sade, como para san Agustín, la existencia del ser humano es una caída en el caos. Para san Agustín, el Verbo representaba la estabilidad, la voz a la que vuelve el ser humano en su desgarre; pero en Sade esa estabilidad no existe y sólo queda la persecución del instante, reconstruido una y otra vez mediante la excitación de la orgía. En otras palabras, en el extravío que genera el orgasmo, el libertino encuentra un respiro que lo saca de la tensión provocada por una existencia que, a su vez, se quiere lograr entender a través de la disertación.

La iniciación de Eugénie se lleva a cabo mediante la repetición de dos acciones: la explicación de algún concepto y la práctica sexual. A lo largo del relato, los personajes ejecutan la diversidad de cuadros sexuales organizados por Dolmancé y repiten de manera constante los orgasmos. Así, por medio de la reproducción continua de las mismas acciones, se materializa un tiempo hierofánico: $:^{15}$

${ }^{15}$ En el Tratado de historia de las religiones Eliade especifica que: “El término ‘tiempo hierofánico’ [...] puede designar el tiempo en que se coloca la celebración de un ritual y que es, por ello mismo, un tiempo sagrado, es decir un tiempo esencialmente diferente de la duración profana a la que sigue. Puede designar también el tiempo mítico, ya sea recuperado por medio de un ritual, ya sea realizado por la repetición pura 
En la religión como en la magia, la periodicidad significa ante todo la utilización indefinida de un tiempo mítico hecho presente. Todos los rituales tienen la propiedad de suceder ahora, en este instante. El tiempo que presenció el acontecimiento conmemorado, repetido por el ritual en cuestión se hace presente es "re-presentado", si así puede decirse, por muy remoto que se lo imagine en el tiempo (Eliade, 1964: 349).

En el ritual iniciático que constituye La philosophie dans le bouoir se pretende restituir un tiempo mítico, pero no se trata de aquél de la eternidad cristiana, fundamentada en la existencia de un ser supremo que organiza el mundo, sino el de un coito originario que localiza la clave del saber universal en el propio cuerpo.

Al hombre de la Ilustración le ha sido arrebatada la creencia en un ser supremo y por lo tanto, el consuelo de una estabilidad eterna. En su lugar se colocó una concepción definida de la naturaleza cuyo conocimiento: "no sólo conduce el mundo de los objetos, sino que se convierte para el espíritu en el medio dentro del cual lleva a cabo su propio conocimiento" (Cassirer, 1932: 54). Más allá de las cuatro verdades de inexistencia que Foucault coloca en el centro de la narrativa sadeana, Eugénie descubre una única verdad que puede ser comprobable: la materialidad del ser humano y, por lo tanto, su individualidad, su isolismo. Esta verdad se corresponde con uno de los principios fundamentales de la filosofía de la Ilustración: "la ley que obedecen los seres singulares no les ha sido prescrita por un legislador extraño, sino que radica en su propio ser y nos es cognoscible totalmente por él" (59).

La restauración del tiempo original que resulta del rito iniciático y la comprensión del ritual hacen posible asir la vida misma, pues: "el tiempo original sirve de modelo a todos los tiempos. Lo que sucedió un día, se repite sin cesar. Basta con conocer el mito para comprender la vida" (Eliade, 1964: 356). Ceder ante el deseo es regresar a la naturaleza, escuchar la voz interior que prescribe la conducta y, en ese sentido, el ser humano abandonado a la persecución y perpetuación del orgasmo, busca la restauración del tiempo primordial, es decir, de la eternidad.

\subsubsection{Más que sadismo, isolismo}

En Aline y Valcour, dentro de una argumentación en torno a la superioridad física del hombre dada por la naturaleza y que por lo tanto debe utilizarse en el acto sexual al infringir dolor en la mujer, se lee lo siguiente: "Je vois cela bien différemment, et ces voluptés doivent être bien tristes, toutes les fois qu'elles ne sont point partagées; l'isolisme m'effraye; je le regarde comme un fléau; je le vois comme la punition de l'être cruel ou méchant, abandonné de toute la terre; il doit l'être de sa compagne, il n'a pas su répandre le bonheur; il n'est plus fait pour le sentir"' (1795: 128). 
En esta segunda acepción, el isolismo define la experiencia sexual que no puede ser compartida. Al principio de la lección, Eugénie afirma que su placer no puede ser transmitido con palabras, y más adelante, cuando Dolmancé se encuentra en medio de una disertación en torno a la inexistencia de impedimentos para llevar a cabo un crimen, por más atroz que éste sea, la joven pregunta:

EUGÉNIE: Mais si l'action nuisait à une très grande quantité d'individus, et qu'elle ne nous rapportât à nous, qu'une très légère dose de plaisir, ne serait-il pas affreux de s'y livrer alors?

DOLMANCÉ: Pas davantage, parce qu'il n'y a aucune comparaison entre ce qu'éprouveront les autres et ce que nous ressentons: la plus forte dose de douleur chez les autres doit assurément être nulle pour nous, et le plus léger chatouillement de plaisir, éprouvé par nous nous touche [...] La source de tous nos erreurs en morale vient de l'admission ridicule de ce fil de fraternité qu'inventèrent les chrétiens, dans le siècle d'infortune et de détresse [...] Mais il est impossible d'admettre cette doctrine! ne naissons pas tous isolés; je dis plus, tous ennemis les uns des autres; tous dans un état de guerre perpétuelle et réciproque? (98-99, las cursivas son propias).

El placer como imperativo es también una fuente del isolismo. Si el ser humano no es capaz de comprender el mundo más que a través de su cuerpo y su individualidad, la ética fundamentada en una metafísica de las costumbres, establecida por Kant, no tiene cabida en el imaginario libertino. En el texto, la sexualidad y con ella las prácticas eróticas que profundizan su experiencia, más que un aspecto de la vida de los hombres, se vuelven imperativo categórico. El isolismo define la incapacidad de otredad del ser humano en la práctica sexual, pues, como remarca Dolmancé, en el extravío de la sexualidad lo único certero es el placer propio. Si la naturaleza ha creado a todos en el aislamiento, no puede existir un mandato que provenga de ella que nos impulse a cuidar de los otros, a preferir la felicidad y el bienestar de los otros por encima de los nuestros. Ésa es la descripción que de manera tradicional se ha hecho del sadismo; sin embargo, el término no engloba la profundidad que posee el isolismo, mucho más adecuado para describir las prácticas sexuales de los villanos de Sade y que, de manera desafortunada, no ha sido rescatado en el léxico.

Si nos apegamos a la tradición filosófica de la época, en particular a lo establecido por Kant en la Fundamentación de la metafísica de las costumbres, los razonamientos de Dolmancé y Saint-Ange, que pretenden establecer una ética con base en el empirismo, caen en un error filosófico fundamental:

Las leyes morales, junto con sus principios, no sólo se diferencian esencialmente, dentro de todo el conocimiento práctico, de todo lo restante en lo que haya cualquier cosa empírica, sino que toda la filosofía moral descansa enteramente sobre su parte pura, y, aplicada al hombre, no toma prestado ni lo más mínimo del conocimiento del mismo (antropología), sino que le da, como ser racional, leyes a priori, las cuales, desde luego, exigen además una capacidad de juzgar aguzada por la experiencia en parte para distinguir en qué casos tienen su aplicación, y en 
parte para provocarles acceso en la voluntad del hombre y energía para la ejecución, pues éste, afectado él mismo con tantas inclinaciones, es ciertamente, capaz de la idea de una razón pura práctica, pero no puede tan fácilmente hacerla eficaz in concreto en su modo de vida.

Una metafísica de las costumbres es, así pues, indispensablemente necesaria, no meramente por un motivo de la especulación, para investigar la fuente de los principios prácticos que residen a priori en nuestra razón, sino porque las costumbres mismas permanecen sometidas a todo tipo de corrupción mientras falte ese hilo conductor y norma suprema de su correcto enjuiciamiento (2010: 111).

Con la categorización del placer individual, Sade no construye una filosofía, más bien profundiza la situación tradicional expuesta en la novela libertina de sus contemporáneos.

El tópico de iniciación es recurrente en los relatos del género, Manon Lescaut, Les égarements du cœur et de l'esprit, Therèse philosophe, Le portier de Chartreux y Les liaisons dangereuses son textos que narran el descubrimiento de sus personajes del mundo amoroso y erótico. De acuerdo con Reichler, en la situación iniciática libertina se repite un patrón que comienza con la idealización amorosa de una joven, continúa con el desengaño y termina con la asunción de un saber. La entrada en el mundo erótico y sexual es, para Reichler, una desacralización del texto bíblico en donde el iniciado se encuentra frente a un nuevo Árbol del Conocimiento que revela un secreto que facilita la inserción social del iniciado: "Toute maîtrise, dit ce secret, est corrompue et corruptrice; toute respectabilité est un leurre; toute croyance une fable" (1982: 105). El conocimiento adquirido, además de ejercer una dura crítica de cualquier tipo de autoridad "place le désir au centre de sa conception de l'homme et de la société, et fait du sexe, objet unique du désir, l'enjeu d'une connaissance réservée" (106). Para Reichler, la desacralización de la mujer en Sade organiza una homologación entre las relaciones intrafamiliares y el macrocosmos cultural e histórico:

La lutte contre l'idéalisation tient une place considérable que les supplices infligés à Madame de Mistival résument symboliquement: violée et infectée, elle est ensuite cossue, en sorte qu'elle bafoue dans sons corps la double inscription mythique dont elle est porteuse: virginité et fécondité. Nulle déréliction, chez Sade, apparemment, puisqu'il n'y a pas d'objet inaccessible, nul besoin non plus d'un achèvement plus heureux. Tout Sade parait voué à développer à l'infini les aventures de la maîtrise, et le pamphlet intitulé «Français encore un effort...», inséré dans la Philosophie rappelle comme une pétition de principe, en termes d'histoire politique, qu'il ne saurait y avoir de demande insatisfaite, de quête prolongée: il prescrit aux citoyens et citoyennes, pour que ce soient enfin concrétisées des mœurs républicains, le devoir de prêter leur corps à quiconque le désire. Et cependant la visée d'une référence idéale, d'une illusion féconde, bref d'une souveraineté imaginaire, ne peut être totalement évacuée. Elle imprime à l'œuvre de Sade un mouvement de balance et confirme, dans le récit libertin d'initiation l'existence d'une incomplétude (108). 
La mujer sacralizada por el cristianismo, vuelta símbolo de virginidad y fecundidad es ahora prostituta negada a la reproducción. El suplicio de Mme. de Mistival es la ordalía que culmina la iniciación de Eugénie y su colocación al final del relato dota de sentido al texto: al coser la vagina infectada de su madre, Eugénie desafía a la autoridad social, a la institución familiar y acepta el deber de entregarse con el único objetivo de dar y recibir placer; nunca con el de perpetuar la existencia del ser humano y sus leyes. Así, el saber libertino es un resultado aparentemente lógico de las ideas de la Ilustración.

Si bien en La philosophie dans le boudoir no existe una historia verosímil de cómo una joven se vuelve libertina, sí hay un relato verosímil y estructurado del rito iniciático que introduce en el mundo a una joven ya predispuesta al libertinaje; el relato de cómo una joven descubre su individualidad dentro de la colectividad social, una iniciación en el culto isolista.

A manera de conclusión, se puede decir que la sexualidad humana desplegada en el texto sadeano resalta la oposición entre los tiempos íntimo y social del ser humano. De acuerdo con Bataille (1957) la prohibición de la sexualidad se encuentra relacionada con el descubrimiento de la mortalidad y la consecuente institución del trabajo. El tiempo del trabajo es aquel que es productivo y da sentido a la vida porque nos aleja del estatismo que representa la muerte; en ese sentido, la sexualidad es un tiempo no productivo que debe ser regulado e incluso evitado. Ahora bien, el erotismo es el aspecto que distingue la sexualidad humana de la animal, pues éste es resultado de la capacidad cognitiva. Pero no toda la actividad sexual del ser humano es erótica, sólo lo es aquella que no se lleva a cabo de manera mecánica. El descubrimiento de la excitación por medio de la estimulación del intelecto moviliza la vida interior, pues en cada acto erótico se cuestiona al ser, quien busca un complemento en el otro. Así como la prohibición sexual surge del descubrimiento de la mortalidad y la institución del trabajo, la violencia reprimida es también resultado de la civilización. Para Bataille muerte y trabajo están estrechamente relacionados. Trabajamos como resultado de la sensación de abismo que produce en nosotros la muerte, y a su vez la muerte es algo horroroso pero admirable:

El mundo del trabajo y la razón es la base de la vida humana; pero el trabajo no nos absorbe enteramente y, si bien la razón manda, nuestra obediencia no es jamás ilimitada. Con su actividad, el hombre edificó el mundo racional, pero sigue subsistiendo en él un fondo de violencia. La naturaleza misma es violenta y, por más razonables que seamos ahora, puede volver a dominarnos una violencia que ya no es la natural, sino la de un ser razonable que intentó obedecer, pero que sucumbe al impulso que en sí mismo no puede reducir a la razón (1957: 44).

Las transgresiones que se llevan a cabo en la orgía sadeana horrorizan no porque se encuentren dentro de la naturaleza, como fallidamente intentan demostrar Dolmancé 


\section{$132 \square$ EL RITO DE INICIACIÓN EN LA PHILOSOPHIE DANS LE BOUDOIR DEL MARQUÉS DE SADE}

y Saint-Ange, sino porque son producto de seres racionales en lucha consigo mismos. El aprendizaje de Eugénie no se limita a establecer la simple búsqueda del placer corporal como motor de la existencia, va más allá, pues en esa búsqueda se inserta una cuestión moral. En el contexto histórico de creación de la obra, el libertinaje existe porque las leyes y los discursos morales hacen de las prácticas sexuales un tabú que amerita la pena de muerte.

La sexualidad que persiguen los personajes de La philosophie dans le boudoir no es la de un animal que busca la reproducción de su especie, se trata de un erotismo complejo que potencia el placer en la medida en que la mente se libera de los límites impuestos por la sociedad y se retrae en sí mismo. El isolismo es libertad y conocimiento, por lo tanto, está relacionado con una cuestión política y religiosa.

\section{Obras citadas}

BARguILlet, François. 1981. Le roman au XVIII siècle. París: Presses Universitaires de France.

BAtAille, George. 1957. El erotismo. Barcelona: Tusquets.

CASSIRER, Ernst. 1932. Filosofía de la Ilustración. México: FCE.

DiDIER, Béatrice. 1972. "Sade et le dialogue philosophique". Cahiers de l'Association Internationale des Études Français, núm. 24. Pp. 59-74.

Eliade, Miercea. 1964. Tratado de historia de las religiones. México: Era. 1958. Nacimiento y renacimiento. El significado de la iniciación en la cultura humana. Barcelona: Kairós.

FouCAult, Michael. 2013. "Conférences sur Sade”. La grade étrangère. À propos de littérature. París: École des hautes études en sciences sociales. Pp. 145-173.

KANT, Imanuel. 2010. Fundamentación de la metafísica de las costumbres. Madrid: Gredos.

REICHLER, Claude. 1982. “Le récit d'iniciation à l'âge classique”. Littérature, 24. Pp. 100-112.

Riceur, Paul. 1985. Tiempo y narración. Configuración del tiempo en el relato histórico. Trad. Agustín NeIRA. Buenos Aires: Siglo XXI.

SADE, Donatien Alphonse François de. 1998. Euvres, tt. I, II y III. París: Gallimard. Bibliothèque de la Pléiade.

1909. L'oeuvre du Marquis de Sade: Zoloé, Justine, Juliette, la Philosophie dans le boudoir, Oxtiern ou Les malheurs du libertinage: pages choisies, comprenant des morceaux inédits. Introd. Guillaume APPOLLINAIRE. París: Bibliothèque des Curieux. 1801. Les crimes de l'amour. Bruselas: Gay et Douce. 1795. Aline et Valcour ou le Roman Philosophique. París: Girouard. 